\title{
Compatibility of the Active Inductance Double Resonance Quartz Oscillator with Q-MEMS Temperature Sensor
}

\author{
Tetsuya Akitsu ${ }^{*}$, Akira Kudo ${ }^{2}$, Tomio Sato ${ }^{1}$ \\ ${ }^{1}$ Human, Environment and Medical Engineering, Interdisciplinary Graduate School of Medicine and Engineering, \\ University of Yamanashi, Takeda 4-3-11, Kofu, Japan \\ ${ }^{2}$ SEIKO EPSON Corporation, Head-Quarter Owa 3-3-5, Suwa, Japan \\ Email: akitsu@yamanashi.ac.jp
}

Received 13 January 2015; accepted 2 February 2015; published 3 February 2015

Copyright $@ 2015$ by authors and Scientific Research Publishing Inc.

This work is licensed under the Creative Commons Attribution International License (CC BY).

http://creativecommons.org/licenses/by/4.0/

(c) () Open Access

\begin{abstract}
Low-frequency double-resonance quartz crystal oscillator circuit was developed with active inductance aiming the quick start-up in the intermittent operation on the sensor circuit and DC isolation using a Q-MEMS sensing crystal HTS-206. Allan standard deviation indicated $5 \times 10^{-12}$, showing short range stability of the sensor circuit sufficient for the ubiquitous environmental sensor network.
\end{abstract}

Keywords

Q-MEMS, Sensing Crystal HTS-206, Active Inductance Double Resonance

\section{Introduction}

Environmental sensing awaits solutions to reduce the electric-power in monitoring under the limitation of the power source. The ubiquitous sensor network is realized with varieties of sensor circuit and a wireless network. The temperature measurement in the environmental sensing is realized by several methods: thermistors, platinum wire or sheet resistor and semiconductor sensor devices. The temperature is measured as the amplitude of low level DC or modulated signals and faces the difficulty which arises from the drift. Q-MEMS crystal temperature sensor can realize high resolution in the sensing of environmental temperature. Direct digital temperature measurements have been developed by several research groups, usint crystal cut LT, SC-cut or equivalent cut [1]-[7]. In a recent work we reported quick start of quartz crystal oscillator. The quick start of the crystal sensor

\footnotetext{
*Corresponding author.
} 
circuit allows intermittent excitation of the sensor system meeting the request for the power management in the environmental sensing. In this work, we aimed several engineering issues: 1) Quick start of low frequency quartz crystal oscillator circuit in the intermittent operation; 2) Reduction of drift by the direct digital measurement; 3) DC isolation between the sensor and the electronic circuit. Tuning fork type Q-MEMS quartz crystal sensor realizes the direct digital sensing of temperature, where Q-MEMS is a combination word of Quartz and MEMS (Micro Electro Mechanical System), a fabrication process offering high performance in a compact package, with $C I$ values as low as those on ordinary-sized crystals. In recent works, double-resonance quartz crystal oscillator was reported for the enhancement of the frequency pulling [8], the mode separation of the multimode quartz crystal resonator [9] [10], and the start-up acceleration of low frequency quartz crystal oscillator circuit [11]. Start-up acceleration of several Mega Hertz is studied by the gain control in the quartz crystal oscillator using a cascade circuit [12]-[14]. Few works treats the acceleration of the start-up of the low-frequency quartz crystal oscillator. We aim to test the conformity of the acceleration scheme with the Q-MEMS crystal sensor. Stability of the oscillation frequency is discussed based on the moving average of the variance determined for the discrete samples following the proto call of the modified Allan standard deviation for moving average of finite length data is employed as the measuring rule of the short range stability [15]-[17].

\section{Design and Analysis of Quartz Crystal Oscillator}

\subsection{Acceleration of the Start-Up of a Quartz Crystal Oscillator}

Figure 1 shows a circuit diagram of the active inductance double resonance oscillator circuit. The initial oscillation is generated by a part this oscillator acting as a CR oscillator, and after the stable oscillation of the quartz crystal resonator current starts, the double resonance is established between the quartz crystal and an active inductance combined with the parallel capacitance resulting in the generation of negative resistance. $\mathrm{IC}_{1}$ and $\mathrm{IC}_{2}$ are CMOS inverter, where CMOS is Complementary Metal Oxide Semiconductor. HTS-206 Q-MEMS temperature sensing crystal is connected through coupling capacitors which are formed between metallic sheets, $20 \mathrm{~mm}$ square in dimension attached on both sides of a Pyrex glass plate. The value is approximately $C_{16}, C_{17}=18 \mathrm{pF}$.

Essential circuit constants $R_{2}, C_{10}$, and $C_{0}$ determines the resonance condition, where $C_{0}$ is the parallel capacitance of the quartz crystal resonator. $R_{2}$ settles the bias in the initial stage of the oscillation. $C_{10}$ stores the ground potential at the activation of the $V_{c c}$ voltage, inserted between the node connecting two inverters. The oscillation frequency is determined by a recharging-time constant $R_{2}$ multiplied by $C_{10}$. Capacitors $C_{2}$ and $C_{3}$ are load capacitors which is necessary for the generation of negative resistance. $C_{5}$ and $C_{6}$ are pass-capacitors between the bus-line and the circuit ground. $C_{0}$ and $C_{1}$ are reserved for the parallel capacitance of the resonator and the series capacitor of the motion arm. The conductance is controlled by negative feedback resistors $R_{f}=R_{3}, R_{4}$, $R_{5}$, and $R_{6}$.

$$
g_{m f}=\frac{g_{m}}{1+R_{f} g_{m}} .
$$

The problem is if the active inductance can generate the negative resistance, and if the negative resistance is large enough to realize the short start-up time. Practical question is the shift of the resonance frequency of the crystal sensor by the series capacitors. Figure 2 shows simplified equivalent circuit-1. CMOS inverter $\mathrm{IC}_{1}$ and $\mathrm{IC}_{2}$ is replaced by two current sources controlled by the gate voltage $V_{\text {in }}$ and $V_{g}$.

Applying Kirchhoff's law, the relations for $I_{\text {out }}$ and $V_{\text {in }}$ are found. $V_{\text {in }}$ is the input voltage of $\mathrm{IC}_{1}$ and $I_{\text {out }}$ is the output current of $\mathrm{IC}_{2}$.

$$
\begin{gathered}
I_{\text {out }}=2 g_{m f} Z_{4}\left(I_{5}+2 g_{m f} V_{\text {in }}\right)=2 g_{m f} V_{g}=2 g_{m f} Z_{4} I_{4} \\
V_{\text {in }}+R_{2} I_{5}+Z_{4} I_{4}=0 \text {, where } I_{4}=I_{5}+2 g_{m f} V_{\text {in }} . \\
I_{5}=-\frac{1+2 g_{m f} Z_{4}}{R_{2}+Z_{4}} V_{\text {in }} . \\
I_{\text {out }}=2 g_{m f} Z_{4}\left(-\frac{1+2 g_{m f} Z_{4}}{R_{2}+Z_{4}}\right) V_{\text {in }}+4 g_{m f}^{2} Z_{4} V_{\text {in }} .
\end{gathered}
$$




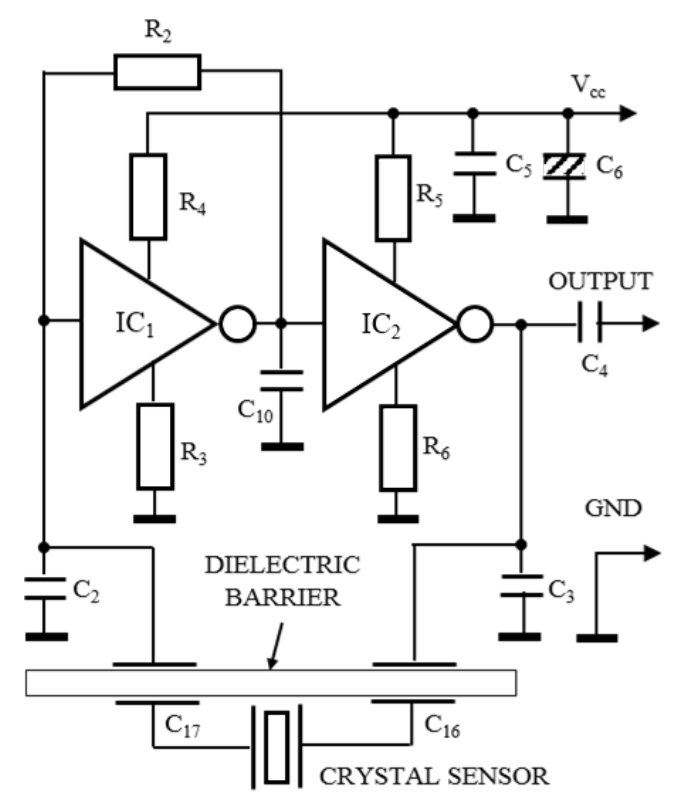

Figure 1. Circuit diagram of the quartz crystal oscillator. Circuit constants: Bias resistors $R_{3}, R_{4}, R_{5}$ and $R_{6}=3.3 \mathrm{k} \Omega$, feedback resistor $R_{2}=3 \mathrm{M} \Omega$, varied for the optimum setting; $C_{2}, C_{3}=10 \mathrm{pF} ; C_{10}=10 \mathrm{pF} ; C_{5}=0.1 \mu \mathrm{F} ; C_{6}=10 \mu \mathrm{F} ; C_{7}=100$ pF; $C_{16}, C_{17}=18 \mathrm{pF}$. Inverter $\mathrm{IC}_{1}$ and $\mathrm{IC}_{2}$ TC7SHU04F; $V_{c c}=3 \mathrm{~V}$. Equivalent circuit constant of the quartz crystal resonator: $f_{1}=40 \mathrm{kHz} ; L_{1}=12,000 \mathrm{H} ; R_{1}=47.6 \mathrm{k} \Omega ; C_{1}=1.326 \mathrm{fF}, C_{0}=803 \mathrm{fF}$.

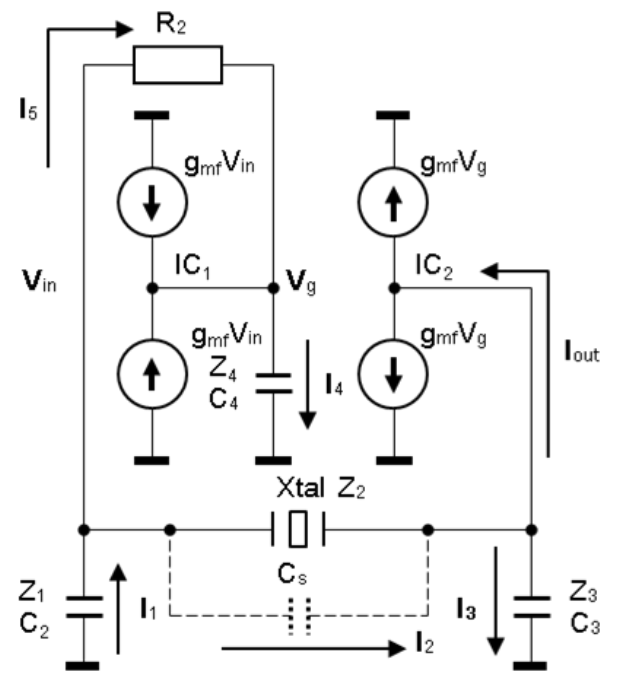

Figure 2. Simplified circuit: equivalent circuit-1.

Solving for the relation between $I_{\text {out }}$ and $V_{\text {in }}$, total conductance $G_{M}$ is found.

$$
G_{M}=\frac{I_{\text {out }}}{V_{\text {in }}}=\frac{2 g_{m f}\left(2 g_{m f} R_{2}-1\right)}{1+\frac{R_{2}}{Z_{4}}} .
$$

Then the following relation is found. Current $I_{2}, I_{3}$ are expressed in the terms of $I_{1}$.

$$
\begin{gathered}
V_{\text {in }}=Z_{1} I_{1} . \\
I_{2}=I_{1}-I_{5}=\left(1+\frac{1+2 g_{m f} Z_{4}}{R_{2}+Z_{4}} Z_{1}\right) I_{1} .
\end{gathered}
$$




$$
I_{3}=I_{2}-I_{\text {out }}=I_{2}-G_{M} Z_{1} I_{1}=\left(1+\frac{1+2 g_{m f} Z_{4}}{R_{2}+Z_{4}} Z_{1}-G_{M} Z_{1}\right) I_{1} .
$$

Rearranging the expression, relation (11) is found.

$$
\begin{gathered}
Z_{1}+Z_{3}-G_{M} Z_{1} Z_{3}=Z_{c}, \quad Z_{c}=R_{c}+\frac{1}{j \omega C_{c}} . \\
Z_{2}+\frac{\left(\frac{R_{2}}{Z_{4}}+1\right) Z_{c}+\left(\frac{1}{Z_{4}}+2 g_{m f}\right) Z_{1} Z_{3}}{\frac{R_{2}}{Z_{4}}+1+\left(\frac{1}{Z_{4}}+2 g_{m f}\right) Z_{1}}=0 .
\end{gathered}
$$

$Z_{2}$ is the impedance of a quartz crystal resonator $\left(Z_{x t}\right)$, and impedance for other components is defined as in (12). The composed impedance $Z_{c c}$ of the active circuit is found, substituting the impedance. From the condition for the non-zero solution of current, the oscillation condition results in (13). The impedance of the circuit is divided into resistive and reactance parts.

$$
\begin{gathered}
Z_{2}=Z_{x t}, Z_{1}=\frac{1}{j \omega C_{2}}, Z_{3}=\frac{1}{j \omega C_{3}}, Z_{4}=\frac{1}{j \omega C_{4}}, \omega_{r}=\frac{1}{C_{4} R_{2}} . \\
Z_{x t}+Z_{c c}=0 \\
Z_{c c}=R_{c c}+j X_{c c}=\frac{R_{c}+\frac{1}{\omega_{r} C_{c}}-\frac{2 g_{m f}}{\omega^{2} C_{2} C_{3}}+j\left(\frac{\omega R_{c}}{\omega_{r}}-\frac{C_{4}}{\omega C_{2} C_{3}}-\frac{1}{\omega C_{c}}\right)}{1+\frac{C_{4}}{C_{2}}+j\left(\frac{\omega}{\omega_{r}}-\frac{2 g_{m f}}{\omega C_{2}}\right)} .
\end{gathered}
$$

The equivalent resistance and the reactance of the circuit are found. Equivalent inductance $L_{c c}$ or capacitance $C_{c c}$ is determined depending on sign of reactance $X_{c c}$.

$$
R_{c c}=\frac{a c+b d}{a^{2}+b^{2}}, X_{c c}=\frac{a d-b c}{a^{2}+b^{2}},\left(L_{c c}=\frac{X_{c c}}{\omega}, C_{c c}=-\frac{1}{\omega X_{c c}}\right) .
$$

Factors " $a$ ", " $b$ ", " $c$ " and " $d$ " are introduced for the simplicity of the expression, where factors " $c$ " and " $d$ " have the dimension of " $\Omega$ " and factors " $a$ " and " $b$ " are dimensionless numbers.

$$
a=1+\frac{C_{4}}{C_{2}}, b=\frac{\omega}{\omega_{r}}-\frac{2 g_{m f}}{\omega C_{2}}, c=\frac{1}{\omega_{r} C_{c}}+R_{c}-\frac{2 g_{m f}}{\omega^{2} C_{2} C_{3}}, d=\frac{\omega R_{c}}{\omega_{r}}-\frac{C_{4}}{\omega C_{2} C_{3}}-\frac{1}{\omega C_{c}} .
$$

$G_{M}$ is separated into real and imaginary parts.

$$
\begin{gathered}
G_{M}=g_{s}+j b_{s} . \\
g_{s}=\frac{2 g_{m f}\left(2 g_{m f} R_{2}-1\right)}{1+\left(\frac{\omega}{\omega_{r}}\right)^{2}}, b_{s}=\frac{\omega}{\omega_{r}} \frac{2 g_{m f}\left(1-2 g_{m f} R_{2}\right)}{1+\left(\frac{\omega}{\omega_{r}}\right)^{2}} .
\end{gathered}
$$

Introducing (13) and (19) into $Z_{c c}$, the impedance of the active circuit is found.

$$
R_{c c}=\frac{g_{s}}{\omega^{2} C_{2} C_{3}}, \frac{1}{C_{c c}}=\frac{1}{C_{2}}+\frac{1}{C_{3}}-\frac{b_{s}}{\omega C_{2} C_{3}} .
$$

Figure 3 shows simplified diagram of equivalent circuit-2 and equivalent circuit-3. The active circuit is indicated with $R_{c c}$ and reactance $C_{c c}$ or $L_{c c}$ depending on the sign. The resonator consists of parallel capacitance $C_{0}$ and the motion arm, $L_{1}, C_{1}$, and $R_{1}$, the equivalent series inductor, capacitor, and resistor respectively. $C_{S}$ is a stray capacitance. Calculating the parallel composition of $C_{0}$ and $C_{s}$ with the active circuit, equivalent circuit-3 


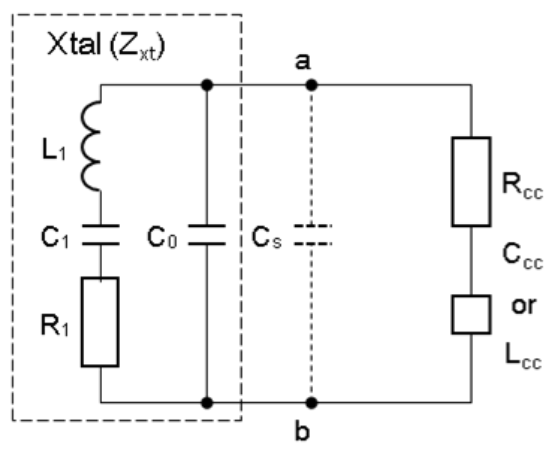

(a)

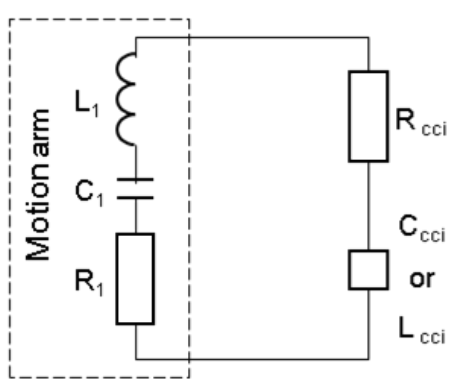

(b)

Figure 3. Simplified equivalent circuit. (a) Equivalent circuit-2; (b) Equivalent circuit-3.

is found. Composed equivalent resistance $R_{c c i}$ and capacitance $C_{c c i}$ are found.

$$
R_{c c i}=\frac{R_{c c}}{\left(1+\frac{C_{0 s}}{C_{c c}}\right)^{2}+\left(\omega C_{0 s} R_{c c}\right)^{2}}, \frac{1}{C_{c c i}}=\frac{\frac{1}{C_{c c}}\left(1+\frac{C_{0 s}}{C_{c c}}\right)+\frac{\left(\omega C_{0 s} R_{c c}\right)^{2}}{C_{0 s}}}{\left(1+\frac{C_{0 s}}{C_{c c}}\right)^{2}+\left(\omega C_{0 s} R_{c c}\right)^{2}} .
$$

Negatively signed capacitance is converted to an active inductance by relation (21).

$$
L_{c c i}=-\frac{1}{\omega^{2} C_{c c i}} .
$$

The denominator of negative resistance $R_{c c i}$ has quadratic dependence on $R_{c c}$. The maximum value of the absolute value is reached at a specific value of $R_{c c}$ determined by $C_{0 s}$ and $C_{c c}$. The following relation is fulfilled.

$$
\left|R_{c c i}\right|_{\max }=\frac{1}{2 \omega C_{0 s}\left(1+\frac{C_{0 s}}{C_{c c}}\right)} \text { at }\left|R_{c c}\right|=\frac{1}{\omega C_{0 s}}\left(1+\frac{C_{0 s}}{C_{c c}}\right) .
$$

The active inductance appears in the vicinity of the resonance frequency, while capacitance $C_{c c}$ is negative. The resonance frequency is determined by $L_{c c}, C_{0 S}$, and the sum of $C_{0}$ and $C_{s}$. In this simplified form, the absolute value of negative resistance $R_{c c i}$ becomes infinitely large, if $C_{c c}$ approaches $-C_{0 s}$ and condition (23) is fulfilled.

$$
C_{c c}=-\frac{1}{\omega^{2} L_{c c}} .
$$

At the resonance frequency determined by $L_{c c}$ and $C_{0 s}$, the absolute value of negative resistance determines the growth of signal. The suppression of negative resistance by inductance $L_{1}$ establishes the stability and inhibitory action against the signal growth. Temperature sensing crystal HTS-206 is a tuning-fork type resonator, 2 $\mathrm{mm}$ in diameter and $6 \mathrm{~mm}$ in length of the exterior size, produced for low power oscillation of $0.1 \mu \mathrm{W}$ typically. Table 1 shows the equivalent circuit constant of the quartz crystal resonator.

Temperature dependence of the crystal sensor is explained in the experimental part. Figure 4 and Figure 5 compare the absolute values of negative resistance $R_{c c}$ and $R_{c c i}$ as functions of frequency. In Figure 4, the absolute value of negative resistance and the active inductance is compared as functions of frequency. The parameter $g_{m f}$ is selected at $4.1 \mu \mathrm{A} / \mathrm{V}$. The active inductance disappears at $55 \mathrm{kHz}$ for $g_{m f}=4 \mu \mathrm{A} / \mathrm{V}$, and $110 \mathrm{kHz}$ for $g_{m f}=$ $8 \mu \mathrm{A} / \mathrm{V}$. The frequency limit is $40 \mathrm{kHz}$ for $g_{m f}=3 \mu \mathrm{A} / \mathrm{V}$ and $110 \mathrm{kHz}$ for $g_{m f}=8 \mu \mathrm{A} / \mathrm{V}$. Larger gain is necessary for the negative resistance and the active inductance.

$$
C_{0}-C_{c c}=0
$$


Table 1. Equivalent circuit constant of the temperature sensing crystal (HTS-206).

\begin{tabular}{ccccccc}
\hline \multirow{2}{*}{ Resonance frequency } & \multicolumn{5}{c}{ Equivalent circuit constant } \\
\cline { 2 - 6 } & $L_{1}$ & $C_{1}$ & $R_{1}$ & $C_{0}$ & $Q_{1}$ \\
\hline $39.992508 \mathrm{kHz}$ & $12 \mathrm{kH}$ & $1.326 \mathrm{fF}$ & $12.6 \mathrm{k} \Omega$ & $803.279 \mathrm{fF}$ & 239,149 \\
\hline
\end{tabular}

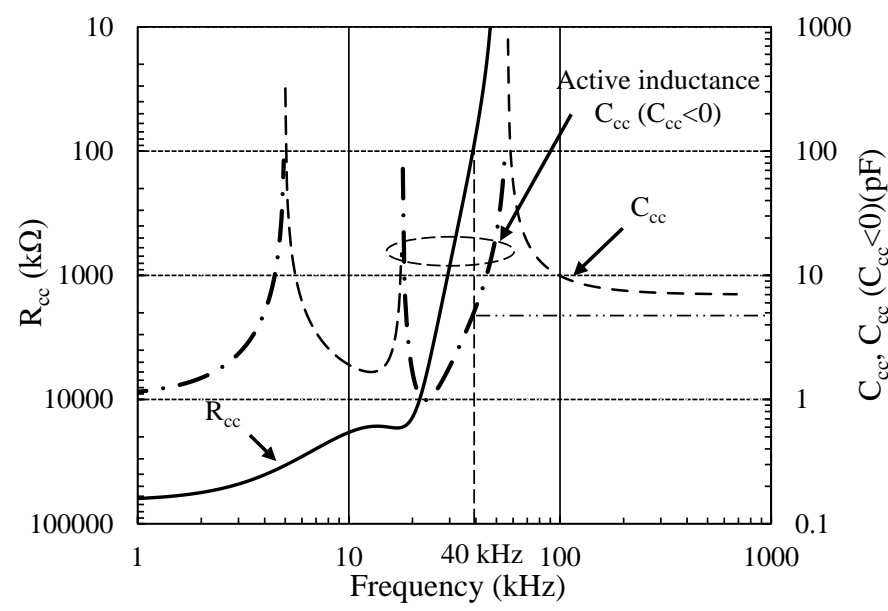

Figure 4. Absolute value of negative resistance $R_{c c}$ and reactance $C_{c c}$ as functions of frequency and gain. Circuit constant: $C_{2}, C_{3}=14 \mathrm{pF}$; $C_{10}=18 \mathrm{pF} ; R_{2}=1.0 \mathrm{M} \Omega, g_{m f}=4 \mu \mathrm{A} / \mathrm{V}$.

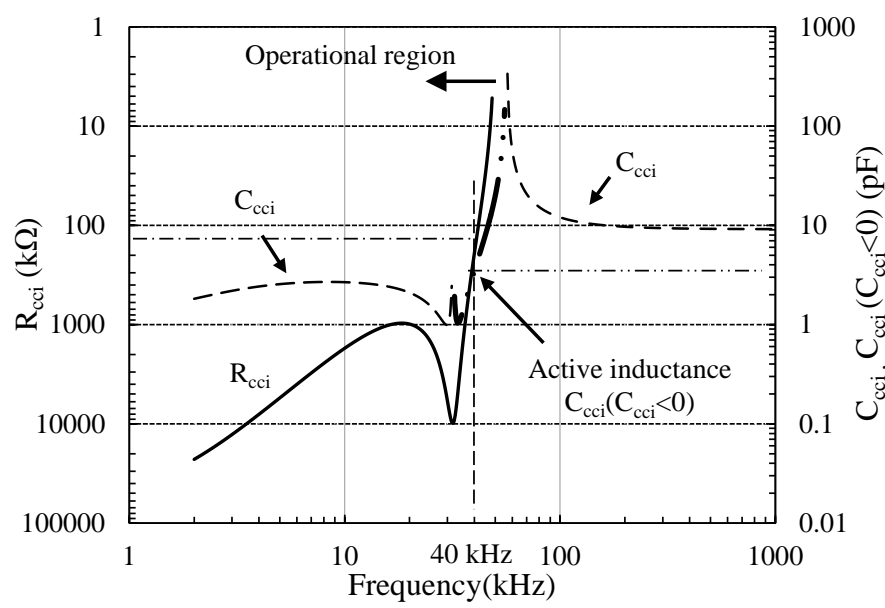

Figure 5. Absolute value of negative resistance $R_{c c i}$ and reactance as functions of frequency and $g_{m f}$. Circuit constant: $R_{2}=1.9 \mathrm{M} \Omega ; C_{2}, C_{3}$ $=14 \mathrm{pF} ; C_{0}=1.14 \mathrm{pF} ; C_{4}=18 \mathrm{pF} ; C_{s}=1 \mathrm{pF} . g_{\mathrm{mf}}=4 \mu \mathrm{A} / \mathrm{V}$.

In Figure 5, the absolute value of negative resistance $R_{c c i}$ and the active inductance is compared as functions of on frequency. The parameter $g_{m f}=4.1 \mu \mathrm{A} / \mathrm{V}$ was optimized for $32.768 \mathrm{kHz}$ realizes the maximum value of negative resistance $R_{c c i}$ approximately of $150 \mathrm{k} \Omega$ and the reactance: $C_{c c i}=-3$ to $-4 \mathrm{pF}$.

Figure 6 shows that the active inductance is generated in narrow range of gain corresponding to the resonance frequency and higher gain is necessarily compared with the case of $32.768 \mathrm{kHz}$, for better performance. The absolute value of negative resistance ranges to $R_{c c}=2 \times 10^{3} \mathrm{k} \Omega$ and the reactance is inductive $C_{c c}=-0.6 \mathrm{pF}$ at $g_{m f}$ $=8 \mu \mathrm{A} / \mathrm{V}$.

In this analysis, the terminal impedance at a - b is expressed with $R_{c c}$ and $R_{c c i}$. The parallel capacitance $C_{0}$ and stray capacitance $C_{s}$ included in the impedance $R_{c c i}$. From relation (22), $R_{c c i}$ becomes infinitely large at $C_{c c}=$ $-C_{0 s}$. This result must be interpreted carefully, because the optimum condition is not realized in the context of 


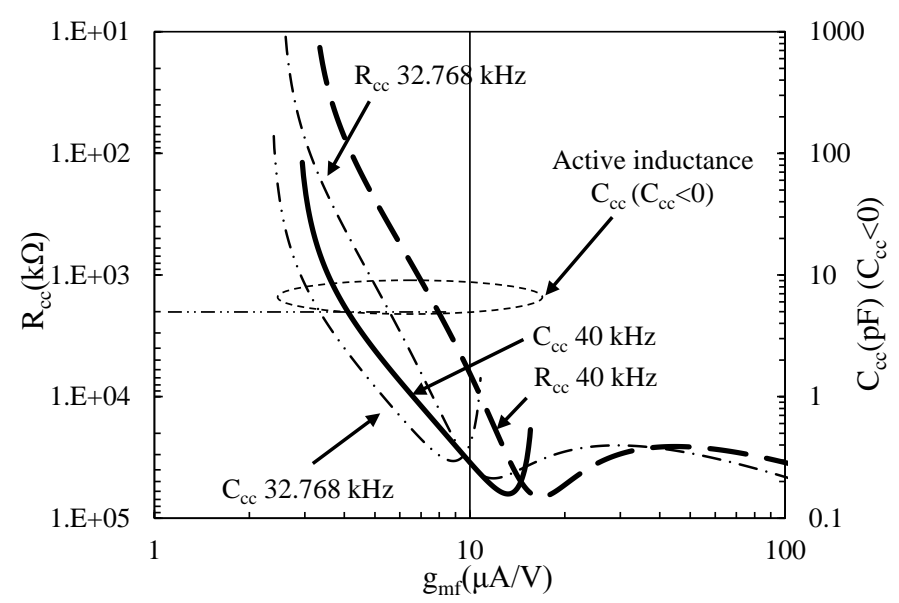

(a)

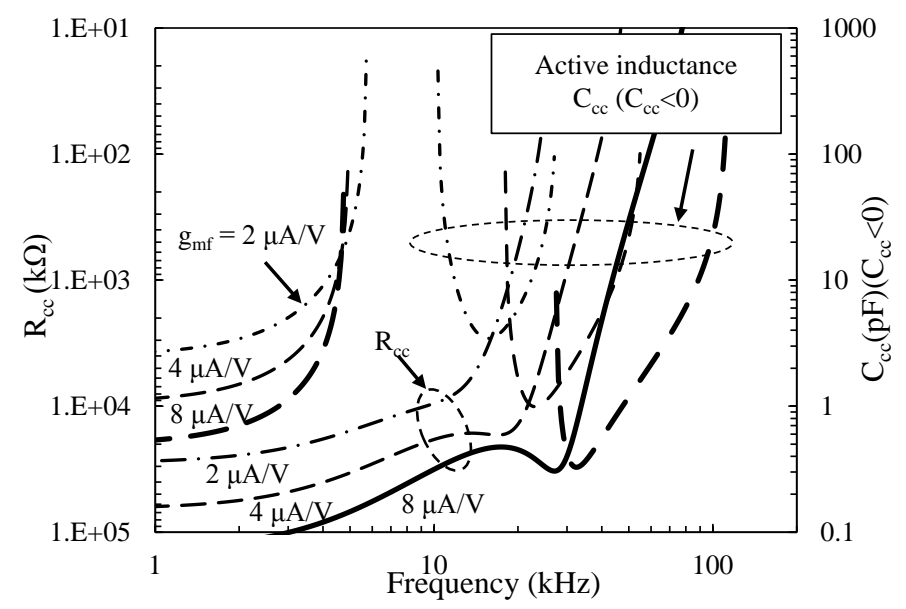

(b)

Figure 6. Comparison of negative resistance for different values of $g_{m f}$ and $f_{r}$. (a) Dependence of negative resistance on $g_{m f}$ for different values of $f_{r}$. Frequency $f_{r}=32.768 \mathrm{kHz}$ and $40 \mathrm{kHz}$. Circuit constants: $C_{2}, C_{3}=14 \mathrm{pF} ; C_{4}=18 \mathrm{pF} ; R_{2}=1.9 \mathrm{M} \Omega$; (b) Frequency dependence of negative resistance for different values of $g_{m f} \cdot g_{m}=2,4$, and 8 $\mu \mathrm{A} / \mathrm{V}$. Circuit constants: $C_{2}, C_{3}=10 \mathrm{pF} ; C_{4}=18 \mathrm{pF} . R_{2}=1.9 \mathrm{M} \Omega$.

the actual circuit design. The idea given in this result is that the active inductance can generate large negative resistance compared to the capacitive region. Actually, $R_{c c}$ is determined under the limitation of the circuit constants and the oscillation frequency. The strength of the oscillation is limited within the linear region of the active circuit.

The curve indicated as $32.768 \mathrm{kHz}$ shows the result calculated using the equivalent circuit constant of a timebase quartz resonator analyzed in Ref. 6. Comparing the dependence on frequency and gain, larger gain is needed for the appropriate design of the active inductance and negative resistance, when the resonance frequency is higher.

\subsection{Modelling of the Start-Up of Oscillation}

Computer simulation was carried out using LTspice IV for Windows (Linear Technology Corporation, 1630 McCarthy Blvd., Milpitas, CA, USA) [18]. Figure 7 shows the circuit layout for the simulation. CMOS inverter $\mathrm{IC}_{1}$ and $\mathrm{IC}_{2}$ are replaced with pairs of complementary MOSFETs (Metal Oxide Semiconductor Field Effect Transistor). Because original $\mathrm{Q}$ is too high for the stable simulation, the motional capacitance and inductance are scaled with the resonance frequency fixed. The motional components do not correspond to the values assigned 


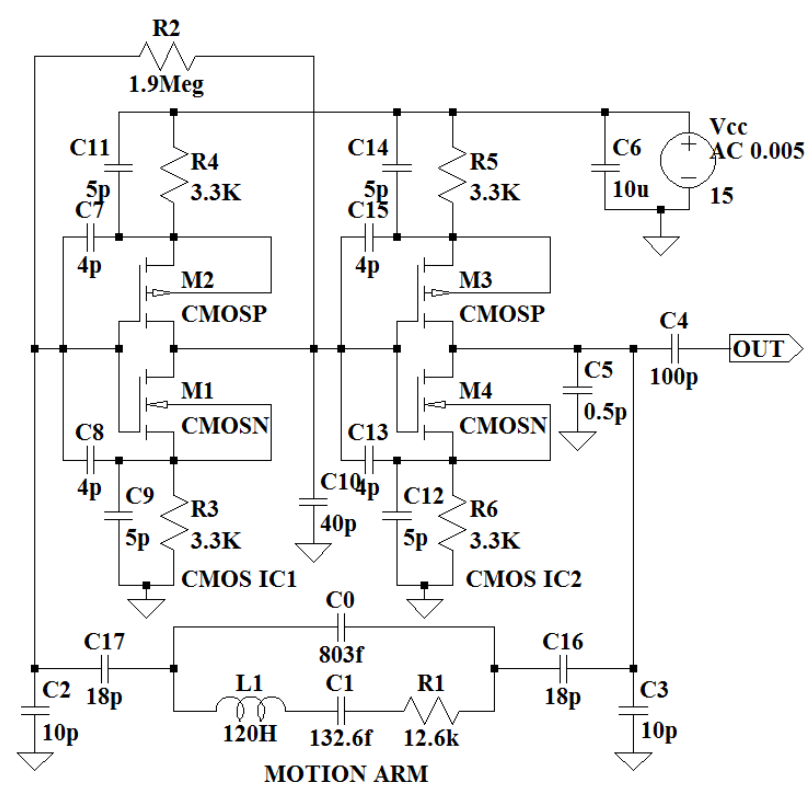

.include "CMOSMODEL.mod"

.tran $00.5 \mathrm{~ms} 0 \mathrm{~s} 0.01 \mathrm{~ms}$ startup

Figure 7. Equivalent circuit for the LTspice simulation. Courtesy of Linear Technology Co.

in the analysis and experiment, neither the delayed connection of the motion arm is considered. When the motion arm is removed, this circuit forms a CR oscillator. The oscillation frequency is determined by the reactance of the parallel capacitance of the quartz resonator and feedback resistor $R_{2}$. Figure 8 shows a typical wave form of the CR oscillator and FFT spectral peak of the initial CR oscillation.

Figure 9 shows the transient excitation of the crystal current with miss-matched frequency setting of the CR oscillation. As the peak of the CR oscillation at $70 \mathrm{kHz}$ is higher than the resonance frequency, phase mismatch can be observed in a few cycles of the initial oscillation. In the optimum setting, the crystal current in the motion arm grows faster, and the oscillation frequency of the oscillator circuit is locked to the resonance frequency within several oscillation cycles.

\section{Experimental Result and Discussions}

The stability of the stable oscillation of the double resonance oscillator is evaluated experimentally. The stability of the oscillation frequency is analyzed with 53230A universal frequency counter (Agilent Technologies, Santa Clara, Ca, USA) synchronized with external rubidium oscillator with long period stability $<2 \times 10^{-11}$ /month and short period stability $<1 \times 10^{-11} / \mathrm{s}$.

\subsection{Stability of the Double Resonance Quartz Crystal Oscillator}

Figure 10 shows the modified two-sample Allan standard deviation as a measure for the short-time frequency stability showing sufficiently high short range stability. The protocol is defined in (25), following IEEE Standard 1139. The frequency of oscillator circuit $f_{k}$ is the discrete sample of oscillation frequency. $\tau$ is the gate time and $\mathrm{k}$ is the sequential number of samples. Dimensionless parameter is defined from frequency deviation normalized by the moving average of finite length data over 10 sequential samples [17]. As a sensor system, the frequency is measured at 100 to $1000 \mathrm{~ms}$ range. Allan standard deviation of $5 \times 10^{-12}$ indicates sufficiently high stability when the sensor is isolated in a constant temperature vessel. The Allan standard deviation shows increase in the range from 1000 to $10,000 \mathrm{~ms}$ indicating the increase in the environmental drift.

$$
\sigma_{y}(\tau)=\left[\frac{2}{M-1} \sum_{k=1}^{M-1}\left(\frac{f_{k+1}-f_{k}}{f_{k+1}+f_{k}}\right)^{2}\right]^{\frac{1}{2}} .
$$



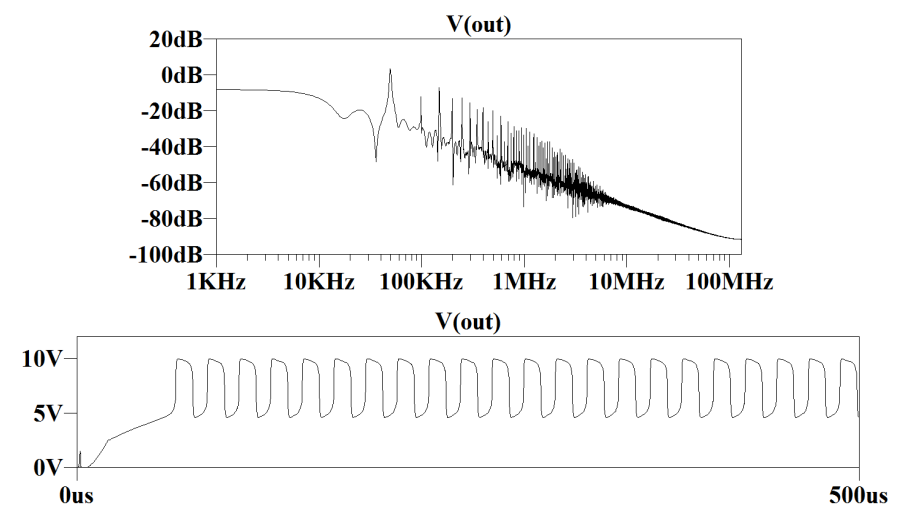

Figure 8. Waveform of the CR oscillator and the FFT spectral analysis $R_{2}=3.0 \mathrm{M} \Omega$.
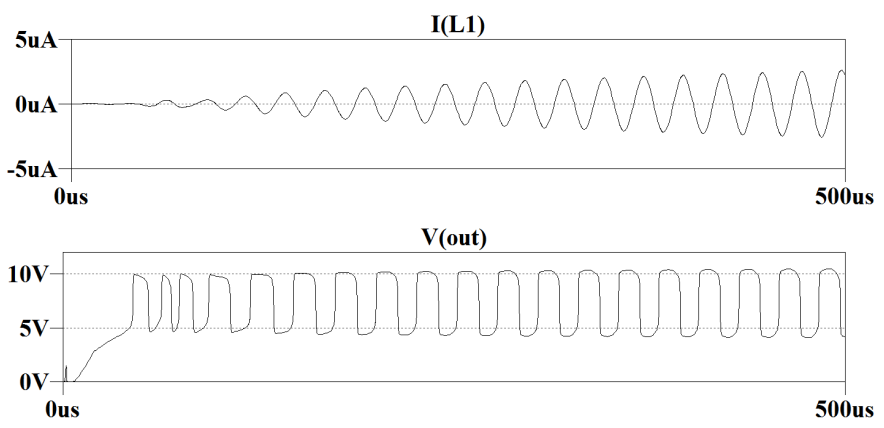

Figure 9. Growth of the crystal current and the frequency locking. Upper track: Current $\mathrm{I}\left(L_{1}\right)$ fowing through motional inductance $L_{1}$. Lower track: Output voltage. $R_{2}=1.9 \mathrm{M} \Omega$.

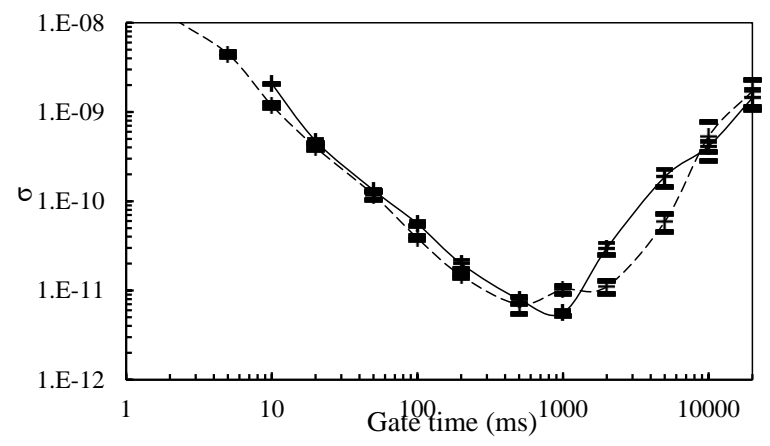

Figure 10. Allan standard deviation showing high short range stability of the crystal oscillator.

\subsection{Temperature Sensing through Coupling Capacitors}

Figure 11 shows the normalized frequency shift as functions of temperature.

In Figure 11, solid line shows the regression curve of the frequency shift in the direct connection normalized with respect to the oscillation frequency at 0 degree C. Cross symbols indicate the frequency shift when the crystal sensor is connected through coupling capacitors: $C_{16}, C_{17}=18 \mathrm{pF}$. Once the regression curve is determined, temperature can be calculated from the oscillation frequency. It is necessary to calibrate the temperature dependence curve must with different standard, because the temperature dependence varies depending on the value of the coupling capacitor. Figure 12 shows the dependence of the resonance frequency on temperature measured by the impedance curve. The active circuit satisfies the oscillation condition for the variation of motional impedance CI over operating temperature. 


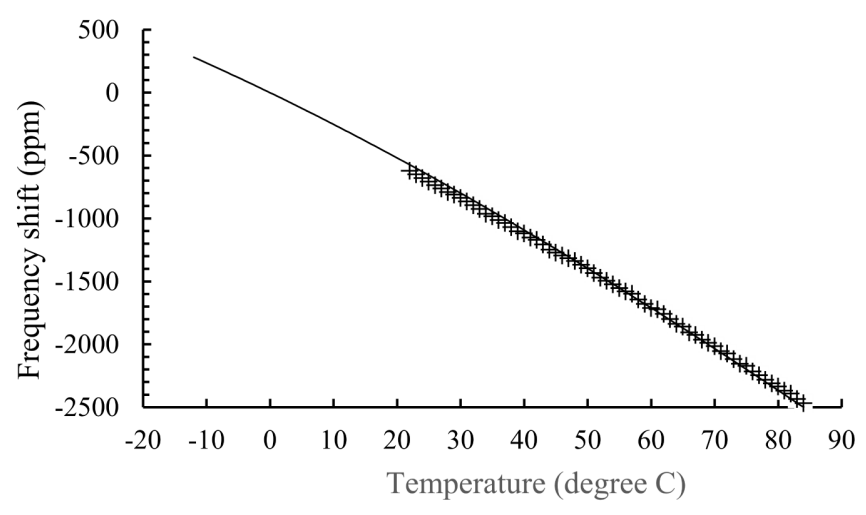

Figure 11. Temperature sensing. Solid line: Direct sensing; $y=$ $2 \mathrm{E}-4 \mathrm{x}^{3}-0.0808 \mathrm{x}^{2}-24.37 \mathrm{x}-0.4608$. Cross symbols: Capacitively coupled sensing, $C_{16}, C_{17}=18 \mathrm{pF} ; \mathrm{y}=-4 \mathrm{E}-05 \mathrm{x}^{3}-0.0495 \mathrm{x}^{2}-$ $24.128 x+72.718$.

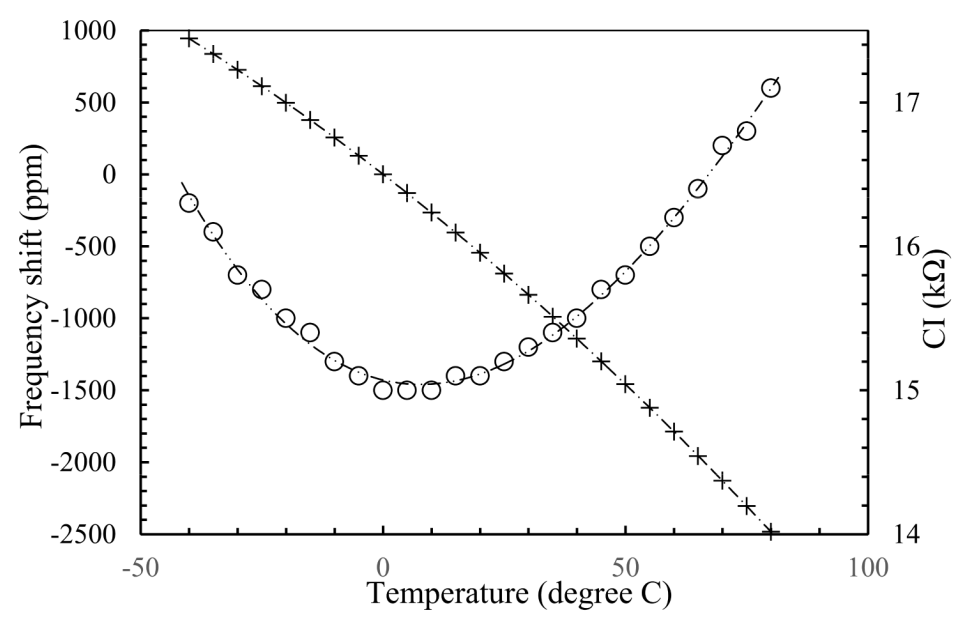

Figure 12. Temperature dependence. Cross symbols: Resonance frequency; Regression curve: $\mathrm{y}=7 \mathrm{E}-7 \mathrm{x}^{3}-0.0623 \mathrm{x}^{2}-26.069 \mathrm{x}+0.7698$. Open circles: CI. HTS-206 sample-F10. Courtesy of SEIKO EPSON Corp.

\section{Conclusion}

Environmental sensing awaits solutions to reduce the electric-power in monitoring under the limitation of the power source. The quick start of the crystal sensor circuit allows intermittent excitation of the sensor system meeting the request for the power management in the environmental sensing. In this work, active inductance double resonance circuit resolved the engineering issues for the quick start-up: 1) Large negative resistance; 2) Low distortion and linearity; 3) Triggering circuit. The quartz crystal oscillator is triggered with a CR oscillator, and transferred to a stable excitation within several period. The maximum negative resistance ranges to $2 \mathrm{M} \Omega$ at specified gain of the active CMOS inverter circuit. The composed reactance of the active circuit negative capacitance $C_{c c}=-0.6 \mathrm{pF}$. Simulation showed the rapid start-up of the oscillation by the energy transfer by the initial $\mathrm{CR}$ oscillation. The oscillation condition was examined by the analysis, the start-up in the computer simulation and examined by the experiment. The stability of the double-resonance oscillator showed short range stability of $5 \times 10^{-12}$ which satisfied the industrial requirement for the resolution of the standard quartz crystal sensor.

\section{Acknowledgements}

The authors acknowledge Mr. Ruan Zheng and Mr. Satoshi Goto for their collaboration in the early stage of this experiment. This work was supported in part by JST A-STEP Contract No. AS251Z01794J. 


\section{References}

[1] Nakazawa, M., Takemae, T., Miyahara, A. and Matsuyama, K. (1985) A Study of Quartz Temperature Sensors Characterized by Ultralinear Frequency-Temperature Responses. IEEE Transactions on Sonics and Ultrasonics, 32, 828834. http://dx.doi.org/10.1109/T-SU.1985.31672

[2] Ueda, T., Kohsaka, F., Iino, T. and Yamazaki, D. (1986) Temperature Sensor Using Quartz Tuning Fork Resonator. The 40th Annual Symposium on Frequency Control, 28-30 May 1986, Philadelphia, 224-229.

[3] Matko, V. and Safsric R. (2009) Major Improvements of Quartz Crystal Pulling Sensitivity and Linearity Using Series Reactance. Sensors, 9, 8263-8270. http://dx.doi.org/10.3390/s91008263

[4] Filler, R.L. and Vig, J.R. (1993) Long-Term Aging of Oscillators. IEEE Transactions on Ultrasonics, Ferroelectrics, and Frequency Control, 40, 387-394.

[5] Matko, V. (2011) Next Generation AT-Cut Quartz Crystal Sensing Devices. Sensors, 11, 4474-4482. http://dx.doi.org/10.3390/s110504474

[6] Walls, F.L. and Vig J.R. (1995) Fundamental Limits on the Frequency Stabilities of Crystal Oscillators. IEEE Transactions on Ultrasonics, Ferroelectrics, and Frequency Control, 42, 576-589. http://dx.doi.org/10.1109/58.393101

[7] Matko, V. and Milanovic, M. (2014) Temperature-Compensated Capacitance-Frequency Converter with High Resolution. Sensors and Actuators A: Physical, 220, 262-269.

[8] Ehara, K., Tanaka, H., Sato, T. and Kanno, Y. (2006) Development of Inductive Quartz Crystal Oscillator Circuit with CMOS Inverter. IEEE International Conference on Systems, Man, and Cybernetics, Vol. 2, Taipei, 8-11 October, 14251430.

[9] Izyan-Ruslan, R., Sato, T. and Akitsu, T. (2012) Voltage Controlled Narrow Band Wide-Variable Range Four Segment Quartz Crystal Oscillator. IEEE Transactions on Ultrasonics, Ferroelectrics, and Frequency Control (UFFC), 59, 564572. http://dx.doi.org/10.1109/TUFFC.2012.2230

[10] Sato, T. and Akitsu, T. (2014) Simultaneous Multimode Oscillation of Stress-Compensated Cut Quartz Resonator with Narrow-Band Wide Variable-Range Quartz Crystal Oscillator. Engineering, 6, 973-988. http://dx.doi.org/10.4236/eng.2014.613088

[11] Sato, T., Kudo, A. and Akitsu, T. (2015) Start-Up Acceleration of Quartz Crystal Oscillator Using Active Inductance Double Resonance and Embedded Triggering Circuit. Journal of Sensor Technology, 5.

[12] Lu, W., Tse, T. and Chan, W.-C. (2006) Crystal Oscillator Circuit Having a Start-up Time Reduction Circuit. US Patent 7034628 B1.

[13] Izumiya, S., Asaki, J. and Adachi, T. (2003) Algebraic Analysis Method of Start-Up Characteristics of Cascade Crystal Oscillator. Electronics and Communications in Japan (Part II: Electronic), 86, 32-43. http://dx.doi.org/10.1002/ecjb.10166

[14] Ulmer, R.W. (1990) Stat-Up Circuit for Low Power MOS Crystal Oscillator. US Patent 4956618.

[15] Barnes, J.A., Chi, A.R., Cutler, L.S., Healey, D.J., Leeson, D.B., McGunigal, T.E., Mullen Jr., J.A., Smith, W.L., Sydnor, R.L., Vessot, R.F.C. and Winkler, G.M.R. (1971) Characterization of Frequency Stability. IEEE Transactions on Instrumentation and Measurement, IM-20, 105-120.

[16] Allan, D.W. (1966) Statistics of Atomic Frequency Standards. Proceedings of the IEEE, 54, 221-230. http://dx.doi.org/10.1109/PROC.1966.4634

[17] IEEE Standard 1139. Definitions of Physical Quantities for Fundamental Frequency and Time Metrology—Random Instabilities, E.E. ISBN: 0-7381-1754-4.

[18] Linear Technology Corporation (2014) LTspice IV for Windows. http://www.linear-tech.co.jp/designtools/software/ 
Scientific Research Publishing (SCIRP) is one of the largest Open Access journal publishers. It is currently publishing more than 200 open access, online, peer-reviewed journals covering a wide range of academic disciplines. SCIRP serves the worldwide academic communities and contributes to the progress and application of science with its publication.

Other selected journals from SCIRP are listed as below. Submit your manuscript to us via either submit@scirp.org or Online Submission Portal.
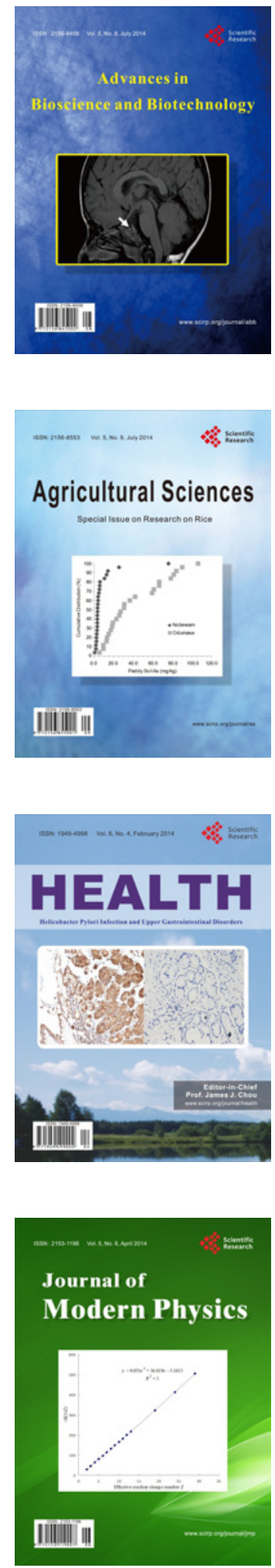
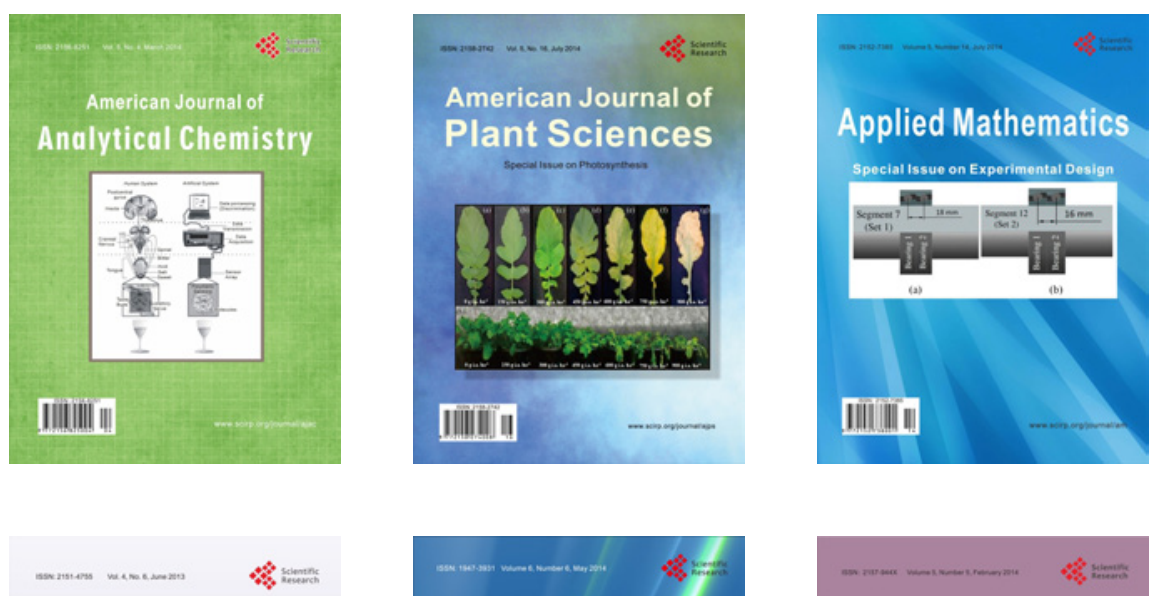

Creative Education
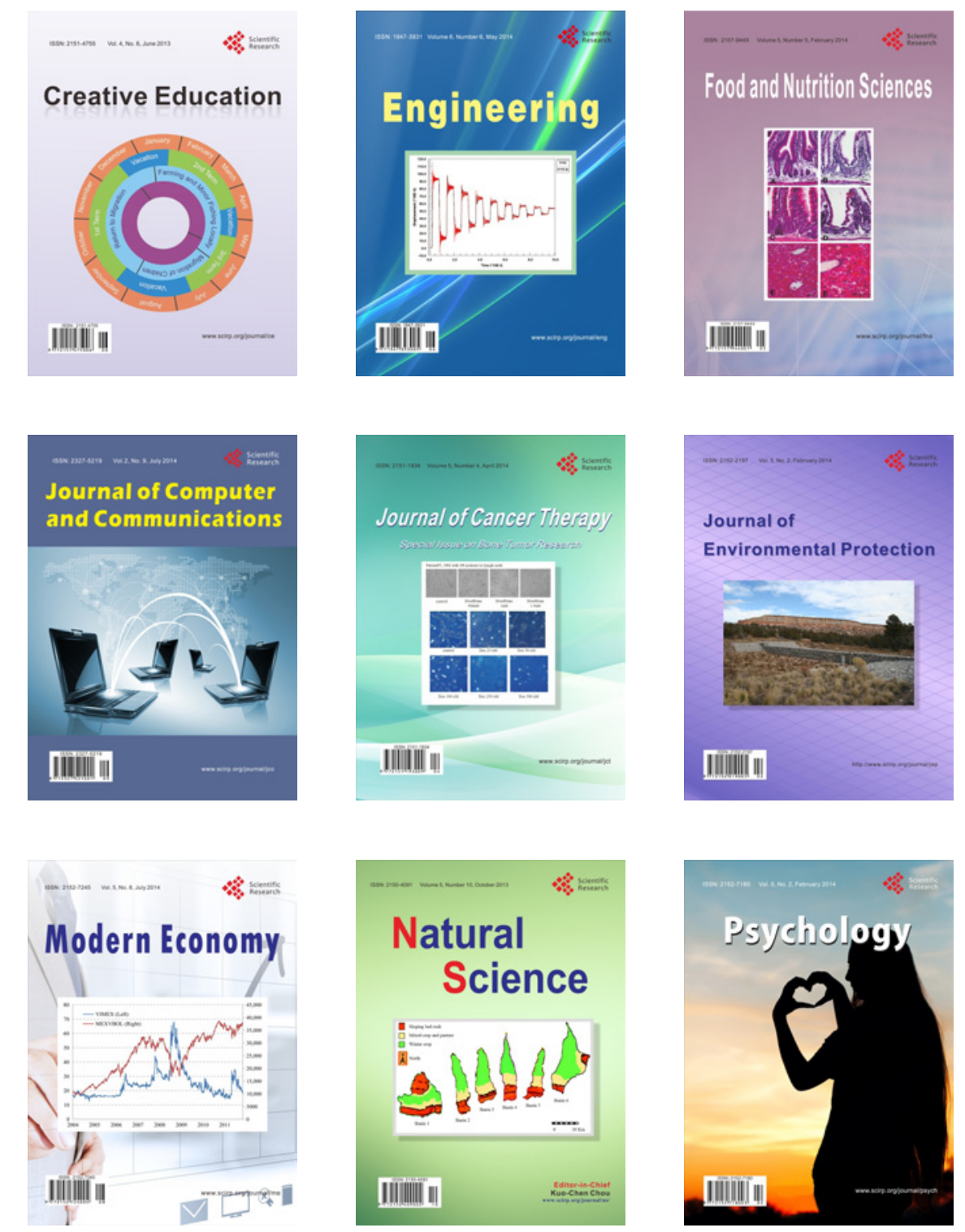